\title{
Application Configuration Selection for Energy-Efficient Execution on Multicore Systems
}

\author{
Shinan Wang ${ }^{\mathrm{a}}$, Bing Luo ${ }^{\mathrm{a}}$, Weisong Shi ${ }^{\mathrm{a}}$, Devesh Tiwari ${ }^{\mathrm{b}}$ \\ ${ }^{a}$ Department of Computer Science \\ Wayne State University \\ $\{$ ez3716,ez6913,weisong\}@wayne.edu \\ ${ }^{b}$ Oak Ridge National Lab \\ tiwari@ornl.gov
}

\begin{abstract}
Modern computer systems are designed to balance performance and energy consumption. Several run-time factors, such as concurrency levels, thread mapping strategies, and dynamic voltage and frequency scaling (DVFS) should be considered in order to achieve optimal energy efficiency for a workload. Selecting appropriate run-time factors, however, is one of the most challenging tasks because the run-time factors are architecture-specific and workload-specific.

While most existing works concentrate on either static analysis of the workload or run-time prediction results, in this paper, we present a hybrid two-step method that utilizes concurrency levels and DVFS settings to achieve the energy efficiency configuration for a workload. The experimental results based on a Xeon E5620 server with NPB and PARSEC benchmark suites show that the model is able to predict the energy efficient configuration accurately. On average, an additional 10\% EDP (Energy Delay Product) saving is obtained by using run-time DVFS for the entire system. An offline optimal solution is used to compare with the proposed scheme. The experimental results show that the average extra EDP saved by the optimal solution is within $5 \%$ on selective parallel benchmarks.
\end{abstract}

Keywords: energy consumption, high performance computing, speedup model, power model, parallel 


\section{Introduction}

The focus of computing has shifted from performance-centered to energy efficiency. As a result, energy-efficient techniques have been adopted across different layers in almost every system, from single chips to large data centers $[1,2]$. Power dissipation and energy consumption are priority concerns when designing computer systems, especially in the High Performance Computing (HPC) field. A recent article suggests that the benefits of the multi-core architecture diminishes as the power constraint on a chip rises [3].

Generally, there are two major factors that affect energy consumption for a specific workload: execution time and average power dissipation. Speedup models are used to describe the benefits introduced by parallel implementations in terms of execution time, while power models are used to estimate power dissipation of a workload. Energy efficiency can be defined as the workload over the required energy, which in turn is equal to $\frac{W}{\left(P_{A I}+\sum_{i}^{C} P_{t}\right) T}$ [4], where $C$ is concurrency level, $P_{A I}$ and $P_{t}$ denote active idle power of a system and average power dissipation of each thread, respectively, and $T$ is execution time. A concurrency level with a thread mapping strategy is referred to a configuration in the rest of the paper.

In-depth analysis of these three factors, $C, P$, and $T$, is necessary to achieve better energy efficiency. For example, a speedup model is usually used to quantify the benefits introduced by parallel computing in terms of execution time [5]. Higher concurrency levels, however, affect power dissipation $(P)$ because not only additional computing units are activated but also the power dissipation of common components on a chip will be shared by more cores. An analytical model is needed to understand the energy efficiency of a workload in a multi-core computing scenario. While allocating a workload to multiple CPUs is an effective way to reduce computation energy consumption, DVFS is usually used to explore slacks during execution to save extra power dissipation [6].

Workload characteristics and micro-architectures have major influence on the three factors. The speedup factor of a workload is closely related to the serial portion of different programs $[7,8]$. In addition, memory boundedness affects the scalability of a workload in the sense that an individual thread or a process competes for off-chip resources with other threads so that concurrency hazards, such as false sharing, might occur [9]. That information cannot be exposed without run-time profiling. On the other side of the spectrum, modern computer systems deploy different mechanisms to 
improve memory performance. For example, Intel processors use Quickpath technology [10] as an implementation of NUMA architecture.

An empirical model is used to predict the configuration as the second step, and voltage/frequency levels of a workload [11, 12] by using Performance Monitoring Counters (PMCs). However, one of the major drawbacks of using an empirical model is architecture dependency, which requires different sets of PMCs to be used for different architectures. Ge et al. propose an energyperformance estimation using an analytical model [13]. However, the model mainly analyzes the behavior of a multi-core based power aware system by case studies. No prediction is used to select the appropriate configuration for each workload.

In this paper, we propose an approach to predict the appropriate configuration of a workload for energy efficiency purposes. First, we propose an analytical speedup model that utilizes PMCs to predict potential speedup of various configurations from two threads execution information. The collected information is used to build the power estimation of various concurrency levels. Once the optimal concurrency level is selected, we apply a run-time DVFS to select an appropriate frequency for each phase. The contributions of this paper include:

- The approach proposed in this paper, rather than training hundreds of PMCs to find the best fit PMCs suite, utilizes only four PMCs based on workload analytical model. The four PMCs are available in most of modern platforms.

- Our model carefully captures the relationship between $C$ (Concurrency Level), $P$ (Power Dissipation), and $T$ (Execution Time), so that we are able to use execution information that using two threads to predict the energy consumption of different configurations on a specific architecture.

- To predict the optimal/near-optimal configuration of a parallel workload, we apply a speedup model. Unlike an empirical model, we reserve the applicability of the model on different architectures by using an analytical model.

- We observe that some parallel applications may have up to $87 \%$ of serial part making the power consumption grow more than benefits obtained by increasing the number of threads. 
The rest of the paper is organized as follows: we start the paper by introducing our observation between workload concurrency level and execution time/power in Section 2; then, we present a two-step prediction model in Section 3, followed by evaluation of the prediction model on a Intel Xeon E5620 platform in Section 4. Related work is discussed in Section 5. Finally, we summarize the paper in Section 6 .

\section{Observation}

In a multi-core or many-core system, the scalability and power dissipation of a workload are closely related to the system architecture and workload characteristics. The execution time could be considered as infinity if no computation unit is involved. As the core number and thread number increase, execution time drops since more computation power is involved. The lower bound of the execution time, however, is limited by two factors, namely the serial portion of the workload and the off-chip resources. As a result, the execution time of a workload approaches its lower bound and even slowly rises as a system allocating more cores to the workload. On the other hand, a system power dissipation increases as the workload occupies more cores. The system consumes idle power if no computation is invoked. Although the system power dissipation increases, it is bounded by the Thermal Design Power (TDP). As a result, the speedup bound and power bound can be modeled using mathematical equations.

Specifically, we model the speedup and power dissipation as a function of the number of threads utilized as Equations 1 and 2, respectively. In particularly, we derive Equation 2 from the logistic function, which has a maximum value $\beta_{1}$ and exponential growth. This scenario corresponds to

the fact that the system power is bounded by the design. $\frac{\beta_{1}}{1+\exp ^{\beta_{2}}}$ represents the system idle power when there is no cores engaged for the workload.

$$
\begin{gathered}
T=f(C)=\alpha_{1}+\alpha_{2} \times C^{\alpha_{3}}+\alpha_{4} \times C^{-\alpha_{3}}\left(\alpha_{3}>0\right) \\
P=g(C)=\frac{\beta_{1}}{1+\exp ^{\beta_{2}-\beta_{3} \times C}}
\end{gathered}
$$

Figure 1 shows the model fitting results for NPB and PARSEC benchmarks. The solid line represents the proposed model while the dots are actual measurements. It is easy to observe that all the benchmarks present a similar pattern, which can be captured by the mathematical models. As the 

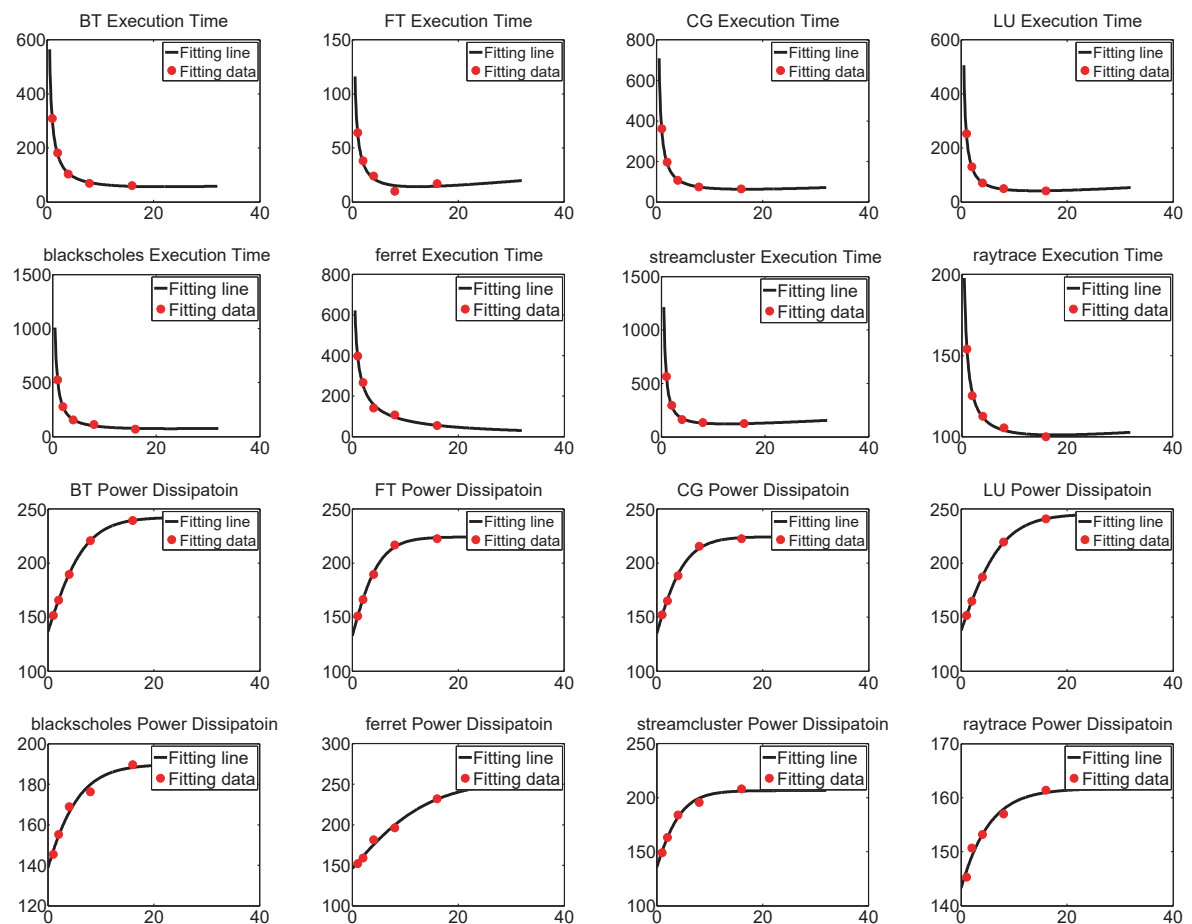

Figure 1: The model fitting of speedup and power dissipation of the workload as a function of concurrency level. The benchmarks are BT, FT, CG, LU, blackscholes, ferret, streamcluster, and raytrace, respectively.

number of threads increases, the workload speedup is limited by the off-chip resources such as cache and memory. On the other hand, the power dissipation, starting from idle power when no thread is running, grows to meet the maximum bound. The mathematical models carefully capture the features of concurrency level and its relationship to the power and speedup. Particularly, the execution time and power dissipation of raytrace benchmark fall into a small range because of the large serial portion of the benchmark. Our model is able to capture this unusual case as well.

We derive models to estimate speedup and power dissipation under various configurations for a workload in order to achieve the best energy efficiency and energy delay product. 


\section{Model Derivation}

In the first step, we focus on concurrency levels $(C)$, power dissipation of the selected concurrency level $(P)$, and execution time $(T)$. By increasing the concurrency level of an application, the average power dissipation increases while the execution time required to finish the same work shrinks. Usually the speedup benefited from a higher concurrency is substantial so that the total energy consumption drops. However, a large number of threads in a system usually results in a competing situation, especially regarding front bus usage. Although NUMA [14] is proposed to solve this problem, off-chip activities affect speedup dramatically. Thread mapping is another technique that is proposed to reduce contention. However, the effects of thread mapping on energy efficiency are uncertain [15, 16]. Although a compact scheme (allocating threads to as less physical processors as possible) generates less power dissipation, this scheme tends to reduce speedup. Scatter scheme (allocating threads evenly to different physical processors), on the other hand, generates higher power dissipation but alleviates contention.

\subsection{Analytical Speedup Model}

In this section, we derive a speedup model for different configurations. The input for the model is collected from the parallel execution with two threads. The output of the model is the speedup factor when applying different thread mapping strategies and concurrency levels. The maximum CPU frequency is always applied in this section. We discuss the model based on the following assumptions and considerations:

- We assume the workload is allocated to a dedicated node and the workload executed directly on the node without virtual machines.

- We measure the workload using two threads and executed on two processor cores. The collected data are the model inputs.

- We apply a simplified memory model that only uses Last Level Cache (LLC) references and misses to quantify the off-chip data accesses.

We assume each workload $W$ is composed of two major parts. One is serial portion, $W^{\text {serial }}$, and the other is parallel portion, $W^{\text {parallel }}$.

$$
W=W^{\text {serial }}+W^{\text {parallel }}
$$




\subsubsection{Serial Portion}

Serial portion of the workload comprises synchronization cost and workload allocation steps; while the former occupies the major portion of a whole $W^{\text {serial }}$. Briefly, synchronization costs include locks [17], barriers, and condition variables. Currently, there is no effective prediction method, as far as we know, to estimate the exact amount of the serial portion of a workload without profiling it in a real world machine. For example, in a recent study [18], Joao et al. designed a new instruction that tracks the amount of cycles elapsed while executing MWAIT instruction in order to identify the serial portion of a parallel program.

Our goal is to predict the synchronization cost of a workload for different system configurations given the information of executing the workload using two threads. Normally threads need to pause and wait for resources or conditions in those synchronization phases. Specifically, threads monitor the data located in some memory addresses to detect any changes. During a monitoring period, a CPU core usually enters the idle state. In order to predict the synchronization cost, our idea is intuitive. The time duration that each thread waits for each other in the two threads execution could be similar to the amount of time each thread waits for others if $n$ threads are used. For example, data in a critical section protected by locks needs to be processed during the execution. It is the same amount of time that is required to process the data no matter how many threads are utilized because of mutual exclusion. We list the average idle time for each thread using different number of threads of two PARSEC benchmarks with native input data, where $i$ denotes the number of processors and $j$ denotes the total number of threads in Table 1. blackscholes is mainly synchronized by barriers; while freqmine uses locks to protect critical sections [19]. It is easy to observe that the average time each thread spending in the idle mode is approximately the same for different configurations. However those idle time is not equally distributed to each thread. We summarize the relationship in Equation 4.

$$
W_{i, j}^{\text {serial }}=W_{2,2}^{\text {serial_avg }} \times j
$$

\subsubsection{Parallel Portion}




\begin{tabular}{|c|c|l|l|}
\hline$i$ & $j$ & $\begin{array}{l}\text { Avg. idle time per } \\
\text { thread for blackholes }\end{array}$ & $\begin{array}{l}\text { Avg. idle time per } \\
\text { thread for freqmine }\end{array}$ \\
\hline 1 & 2 & 28.83 & 4.33 \\
\hline 1 & 4 & 29.23 & 4.41 \\
\hline 2 & 2 & 30.17 & 4.31 \\
\hline 2 & 4 & 29.28 & 4.18 \\
\hline 2 & 8 & 28.89 & 5.30 \\
\hline
\end{tabular}

Table 1: Average idle time for each core using different configurations.

The total amount of workload can be expressed in several ways based on each stage in a pipeline, for instance, $W^{\text {parallel }}=$ total_issue_cycles

+ total_issue_stall_cycle or $W^{\text {parallel }}=$ total_active_execute_cycles

+total_execute_stall_cycle. At the issue stage, stalls mainly stem from instruction cache misses, resource (such as registers) unavailability and other activities, which result in no instruction being allocated to the Reservation Station (RS). These activities do not exhibit a strong relationship to the selected concurrency level. While total_execute_stall measures the delay that is taken to prepare the data for instruction execution.

Part of $W^{\text {parallel }}$ is independent of the concurrency level and the thread mapping strategy. Computation load belongs to this part. We denote this part as $C$. Another part changes significantly mainly because of memory subsystems, which is denoted as $M$. The unit used in the model is cycles.

$$
W^{\text {parallel }}=C+M
$$

The computation load, $C$, once a different concurrency level and thread mapping strategy are applied, can be evenly distributed to the newly assigned threads. The total workload becomes $W_{i, j}^{\text {parallel }}$ and the computation load becomes $C_{i, j}$. The execution time of $C_{i, j}$ part can be reduced to $\frac{2 \times C_{2,2}}{j}$ for each thread. Basically, we use $C_{2,2}$ to estimate the portion of the workload that has been sent to different executing ports on processor units ${ }^{1}$ under the circumstance that all the operands of each instruction are ready for execution. $C_{2,2}$ counts the total time spent on these executions. It is worth noticing that

\footnotetext{
${ }^{1}$ For example, there are 6 different ports on Intel Microarchitecture Code Name Westmere, where port 0,1 , and 5 handle integer arithmetic, SIMD, integer shift, FP multiply and FP divide Uops and Port 2, 3, and 4 handle the load and store Uops[20].
} 


\begin{tabular}{|c|c|c|c|}
\hline$i$ & $j$ & Eexcuted_Cycles for bt.A & Eexcuted_Cycles for ft.B \\
\hline 1 & 1 & 205174317772 & 168308642166 \\
\hline 1 & 2 & 205842270932 & 169576463590 \\
\hline 1 & 4 & 210569866603 & 174047613387 \\
\hline 2 & 2 & 208036560725 & 173953520506 \\
\hline 2 & 4 & 210757446738 & 175101409435 \\
\hline 2 & 8 & 214207223107 & 185063427379 \\
\hline
\end{tabular}

Table 2: $C_{i, j}$ of bt.A and ft.B benchmark.

\begin{tabular}{|c|c|c|c|}
\hline$i$ & $j$ & Stall_Cycles for bt.A & Stall_Cycles for ft.B \\
\hline 1 & 1 & 24536512493 & 18113150861 \\
\hline 1 & 2 & 27213960868 & 31063082823 \\
\hline 1 & 4 & 39855366148 & 81392657035 \\
\hline 2 & 2 & 21026377128 & 23548254140 \\
\hline 2 & 4 & 33466947535 & 42809740979 \\
\hline 2 & 8 & 55621254767 & 110260939317 \\
\hline
\end{tabular}

Table 3: $M_{i, j}$ of bt.A and ft.B benchmark.

thread mapping strategies and different concurrency levels do not affect the computation workload in the analytical model.

We measure $b t$ and $f t$ from NPB benchmark suite to verify the idea. We sample PMC UOPS_EXECUTED_ACTIVE_CYCYCLES (E_Cycles for short), whose event number and umask is $0 \times \mathrm{BB} 1$ and $0 \times 3 \mathrm{~F}$ respectively, using different configurations. The results are shown in Table 2. It is clear that the even though the concurrency level and thread mapping strategy changes, the E_Cycles only varies at most $4 \%$ and $9 \%$ for bt.A and ft.B, respectively.

Changing configurations affects $M_{i, j} . M_{i, j}$ part can be interpreted as the total time used for preparing execution on each unit. Different configurations introduce varying amount of $M_{i, j}$. Even though $M_{i, j}$ is shared by $j$ threads, the total amount of $M_{i, j}$ is related to the specific architecture and is the one that needs to be predicted for each pair of $i$ and $j$. Table 3 illustrates the $M_{i, j}$ part of bt.A and ft.B benchmarks, which is referred as Stall_Cycles. It is calculated by subtracting the Execute_Cycles from the total number of cycles required to finish the workload. As much as $600 \%$ difference can be observed for the benchmark if a different configuration is applied. 
The goal of the prediction model lies on predicting the $M_{i, j}$ for a given $M_{2,2}$. One of the most significant resources stalls is off-chip cache accesses. In a NUMA architecture, a 40 cycles to at most 300 cycles penalty can be triggered in order to access an off-chip L3 cache, while accessing memory triggers $60 \mathrm{~ns}$ to $100 \mathrm{~ns}$ delay [21]. Although the exact number may vary, offchip data accesses become one of the major root causes of execution stalls. As a result, in order to predict $M_{i, j}$, we record LLC reference and LLC miss rates as the input of the model. The unit of them is counts per sec, which shows the approximate demand of LLC and memory accesses during a fixed interval. The prediction is shown in Equation 6, where we denote LLC references and misses as $L L C \_R$ and $L L C \_M$ respectively. Stall_Cycles is referred as Stall for short.

$$
\begin{aligned}
M_{i, j}= & \alpha_{i, j} \times L L C_{-} R_{2,2}+\beta_{i, j} \times L L C_{\_} M_{2,2} \\
& +\operatorname{Stall}_{2,2} \times \gamma_{i, j}+\epsilon_{i, j}
\end{aligned}
$$

In Equation 5, the percentage of $C_{2,2}$ and $M_{2,2}$ is pre-determined by the workload while in Equation 6, the prediction result of $M_{i, j}$ is determined by both the system architecture and the workload itself. Combine Equation 5 and 6 , we obtain Equation 7.

$$
\begin{aligned}
W_{i, j} & =C_{i, j}+M_{i, j} \\
& =\frac{C_{2,2}}{i}+\frac{\alpha_{i, j} \times L L C_{-} R_{2,2}}{i} \\
& +\frac{\beta_{i, j} \times L L C_{-} M_{2,2}+\text { Stall }_{2,2} \times \gamma_{i, j}+\epsilon_{i, j}}{i}
\end{aligned}
$$

In order to obtain $\alpha_{i, j}$ and $\beta_{i, j}, \gamma_{i, j}$, and $\epsilon_{i, j}$, a set of training benchmarks can be used. These parameters are determined by the system architecture, which can be characterized by a set of carefully designed training benchmarks. We modified the memory mountain benchmark [22] by adjusting stride and working set sizes. By changing the stride, we are able to obtain different $L L C_{-} R$ and $L L C_{-} M$ values. Then, we run the modified memory mountain benchmark using different number of threads and thread mapping strategies. PMCs information is collected during each execution. In addition, system power dissipation information is recorded. A Linear Regression model is used to train the model parameters. 
We observed that there are two categories to consider based on the training results. The first category exhibits less data demand that the contention on LLC and memory is limited. The other category shows strong contention by increasing the concurrency level. Two groups are separated by using a predefined threshold value of $L L C \_R$. The rationale behind this scenario is that before the throughput reaches the limit of LLC and memory bandwidths, the relationship between $L L C_{-} R, L L C_{-} M$ and $M_{i, j}$ exhibits differently before and after they reach the limits. We utilize the Minimum Absolute Error to determine the threshold in the training set. Four parameters are obtained for each configuration. We set the threshold, $\delta$, to to 2360000 references/sec based on $L L C \_R$ on the target platform to distinguish each category.

\subsection{Power Model}

By changing concurrency level and mapping strategy, system power dissipation changes as well. In order to achieve a better energy efficiency configuration, a power model describing power dissipation using different configurations is needed. To be clear, the power dissipation that is referred to in this subsection is average power dissipation.

In order to predict the power dissipation of each configuration, we extend the methodology presented in [23]. The previous work predicts the runtime CPU power on multi-core systems using PMCs as input. However, it requires the run-time data collection. In this case, the model input is execution information using two threads and the expected output is average power dissipation of each different configuration.

In the power model, we assume the CPUs do not enter deeper sleep modes but $C 0$. We denote idle power as $P^{b}$ and dynamic power as $P^{d}$. In this paper, we only consider the dynamic power contributed from the CPU and the memory. There are various works that study about the relationship between CPU power and PMCs [24, 25, 23, 26]. Instruction per Cycle (IPC) or UOPs per cycle shows a strong linear relationship to CPU power dissipation. We continue to use this relationship to estimate power dissipation. Instruction_Issued is used in the model instead of Instruction_Required because some instructions are issued and executed but discarded in a pipeline of an OOO execution unit. Regarding off-chip cache and memory power dissipation, $L L C_{-} R$ and $L L C_{-} M$ are introduced to estimate the demand of accesses to LLC cache and memory subsystems. Equation 8 shows the overall estimation for dynamic power. 


$$
\begin{aligned}
P_{i, j} & =P^{b}+P^{d} \\
& =P^{b}+I P C_{2,2} \times a_{i, j}+L L C_{-} R_{2,2} \times b_{i, j} \\
& +L L C \_M_{2,2} \times c_{i, j}+d_{i, j}
\end{aligned}
$$

The power dissipation of the configuration using $i$ processors and $j$ threads is determined by four newly introduced coefficients, $a_{i, j}, b_{i, j}, c_{i, j}$, and $d_{i, j}$ The four parameters are determined by system architecture and can be trained for each pair of $i$ and $j$. However, the $I P C, L L C_{-} R_{2,2}$, and $L L C_{-} M_{2,2}$ are related to the workload characteristics.

In order to obtain $a_{i, j}, b_{i, j}, c_{i, j}$, and $d_{i, j}$, we modified the Memory Mountain Benchmark [22] by adding a computation part after each data item is retrieved. By adjusting the computation load, the stride, and the working set size in the training benchmark, various combination of IPC, $L L C_{-} R$, and $L L C_{-} M$ values can be obtained. For example, setting workload set size to be limited to fit in L3 cache, configuring the stride value to pass L2 cache, and adding a computation part to occupy a reasonable portion in the whole workload, we can generate high $I P C$ and $L L C \_R$ values while maintaining low $L L C_{-} M$. The training process is similar to the speedup model training. Once $a_{i, j}, b_{i, j}, c_{i, j}$, and $d_{i, j}$ are trained for the target platform, the power model is able to predict the power dissipation of another configuration.

The total energy consumption of a workload using various configurations can be calculated as follows:

$$
E_{i, j}=\int P_{i, j} \mathrm{~d} t=P_{i, j} \times W_{i, j}
$$

\subsection{Run-time DVFS}

In the above sections, we describe a static off-line prediction model that uses workload information and system architecture parameters to obtain the speedup factor and power dissipation of different configurations. Applying DVFS [27, 28], we can tune the the power dissipation of CPU at run-time. The CPU frequency is referred as an item in the configuration of a system [11]. However, the proposed method uses DVFS as a run-time knob to tune the results generated from the analytical model described in the previous section because of the following reason: if CPU frequencies are combined with concurrency levels and thread mapping strategies as configurations, the number 
of configurations needed to be considered in an analytical model is huge in a modern multi-core system. For example, on an Intel E5620 platform, there are eight different concurrency and thread mapping configurations (do not consider Intel Hyper Threading technology). If eight frequency levels are considered, the system will generate a total of 64 different configuration settings. The calculation overhead is considerable if we apply it at run-time. Therefore, DVFS is used as the second step to gain further energy savings.

The rationale of tuning DVFS is reducing power dissipation of a system when the workload enters memory bounded phases [29,30]. The primary goal of this step is to reduce run-time power dissipation at the minimum cost of performance degradation.

The first part of the run-time prediction is to classify each phase of a workload to different categories. The second part is the prediction algorithm. In our approach, each phase can be abstracted using a vector of features denoted as $V=\left[v_{1}, v_{2}, \ldots, v_{n}\right]$. Even for the finest grained phase detection mechanism, each phase is combined with computation and memory accesses. Hence, memory boundedness is related to the percentage of computation and data-accesses in a phase. Moreover, the phase duration usually maintains at the range of $10 \mathrm{~ms}$ to $500 \mathrm{~ms}$ [21]. In order to approximate the percentage of off-chip data accesses in one phase, we derive a method similar to the one that is used to predict speedup factors: Stall_Cycles and Execute_Cycles. The percentage of Stall_Cycles in the total elapsed cycles can represent the memory boundedness in some sense and is one of the items in the feature vector used to identify memory-boundedness. However, off-chip memory accesses are not the only source of Stall_Cycles. In order to compensate this phenomenon, we use LLC references and misses as the second and third item in the feature vector. To summarize, the feature vector contains $\frac{\text { Stall_Cycles }}{\text { Total_Cycles }}$, $L L C_{-} R, L L C_{-} M$. We use normalized values of the three elements.

Once the feature vector is determined, we use the modified Memory Mountain Benchmark to generate a set of training samples. We record the values of the three items in the feature vector for each training sample along with the frequency that generates the highest power dissipation deduction over IPC deduction ratio. This way, the training benchmarks can be used to illustrate the maximum power saving with minimum performance degradation. The desired frequency value and the feature vector are collected. Then, all the training cases are grouped into different classes according to its optimal frequency value. In order to predict the behavior of phases, we use k-nearest neighbor algorithm $(k=5)$ to determine different phases. Eu- 
clidean distance is used to determine the similarity between the current phase and the stored training phases. In order to predict the next phase, we use a simple last-value prediction algorithm in runtime to reduce the run-time overhead. The algorithm predicts the very next phase will be the same as the current phase.

\section{Evaluation}

\subsection{Implementation}

The proposed prediction model is implemented with two parts. The first one is the static prediction model that collects execution information using two threads to predict the desired concurrency level and thread mapping strategy. The other one is a run-time phase detection model that dynamically changes CPU frequencies. The parameters in the model are hard coded in the program. We use the perf tool, which is available from Linux kernel system call, _NR_perf_event_open() to sample PMCs values. Perf tool becomes available from Linux kernel 2.6.31 [31].

We collect system information for each core, including idle time for each core from PROC file system. Two Architectural Performance Events are sampled at run-time, which include LLC references, and LLC misses. One of the advantages of using architectural performance events is that they behave consistently across different micro-architectures. Two other event counters in the proposed model, UOPS_EXECUTED_ACTIVE_CYCYCLES and UOPS_ISSUED.ANY are implemented to be architecture-specific. However, these events are supported by most of the recent architectures, which makes our model extensible. To set the configuration after prediction, we utilize omp_set_num_threads() provided by the OpenMp library. sched_setaffinity() system call can be used to change different thread mapping strategies given a CPU topology. The second step works as follows: as a workload starts execution, we sample the PMCs at intervals of $500 \mathrm{~ms}$. The collected values are used to predict the next phase of the execution and set the corresponding CPU frequency. The cpufreq subsystem is used to set different DVFS settings. Xeon E5620 supports one frequency for each processor. We disable Hyper-Threading feature. However, prefetching is enabled. The hardware events selected in the model do not count LLC references and misses due to prefetching, while other events, which are UNC_L3_MISS.ANY (0x309) and $U N C \_L 3 \_H I T . A N Y(0 \times 308)$, count all the L3 cache accesses. 


\begin{tabular}{|c|c|c|c|}
\hline Component & Value & Component & Value \\
\hline CPU & Intel Xeon E5620 & Frequency & $2400 \mathrm{MHz}$ \\
\hline Microarchitecture & Nehalem & Number of sockets & 2 \\
\hline Processor core & Westmere-EP & Num of cores per chip & 4 \\
\hline L1 cache & $\begin{array}{c}4 \times 32 \mathrm{~KB} \text { I cache } \\
4 \times 32 \mathrm{~KB} \text { D cache }\end{array}$ & $\begin{array}{c}\text { Num of threads } \\
\text { per chip }\end{array}$ & 8 \\
\hline L2 cache & $4 \times 256 \mathrm{~KB}$ & Total num of threads & 16 \\
\hline L3 cache & $12 \mathrm{MB}$ & Kernel version & Linux 2.6 .31 \\
\hline
\end{tabular}

Table 4: System specification.

\subsection{Experiment setup}

We conduct the experiments on an Intel Xeon E5620 server. The specification is listed in Table 4. There is a total of eight frequencies available, from $1600 \mathrm{MHz}$ to $2400 \mathrm{MHz}$. We use NPB and PARSEC benchmark suites to conduct the evaluation. In order to measure the energy consumption of the workload on the system, we connect a power measurement device, Watt's Up Pro, between the power outlet and the server. Watt's Up Pro is able to record power dissipation of the entire system at a frequency of $1 \mathrm{~Hz}$. It is connected to the system with a serial port. Watt's Up averages power measurements inside one second interval, so that it is reasonable to use power readings and execution time to calculate total energy consumption.

\subsection{Speedup Model Evaluation}

In this section, we evaluate the accuracy of the proposed speedup model. The input of the model is the information collected from the execution using two threads of the workload. The output is the estimated execution time of different configurations.

Figure 2 shows the serial portion of a workload in terms of absolute execution time and percentage to the entire execution time using eight threads. All of the tested benchmarks from NPB demonstrate less than $2 \%$ serial portion; while PARSEC benchmark suite contains various serial execution phases, ranging from $3 \%$ to $87 \%$ of total execution time. The serial portion limits the speedup that can be achieved for raytrace benchmark, particularly.

Regarding the parallel part, in order to obtain a more accurate prediction, we separate the workload into different categories and train benchmarks for each category. This way, we can obtain the parameters described 


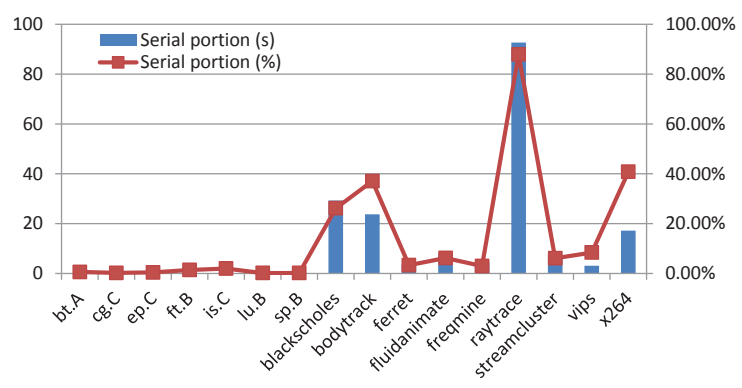

Figure 2: Serial portion of the workload

in Equation 6 as shown in Table 5. Conf refers to the concurrency level and thread mapping strategy used. Ctgy stands for the categories mentioned in Section 3.1. The threshold $\delta$ is set to be 2362346 references/sec. $\gamma$ is used to describe the stall portion. In the case that all four cores are used, namely configuration $(1,4)$ and $(2,8)$, we can observe that the serial setting does not contribute much to the predicted results as other configurations, such as $(1,2)$. As the configuration becomes complicated, the architectural meaning of each parameter diminishes. The reason is because our model only samples the activities of a small portion of the whole system to maintain its applicability. In addition, there are only at most four performance event registers on the state of the art micro-architectures [20], which means only four events can be simultaneously sampled without multiplexing counter registers. Our model uses all four counters, namely LLC references, LLC misses, UOPS_EXECUTED_ACTIVE_CYCYCLES, and UOPS_ISSUED.ANY. The total UnHalted Cycles are calculated from execution time and CPU frequencies.

Table 6 illustrates the percentage of stall cycles in the two thread execution of each benchmark. The percentage of stalls is one of the workload characteristics we discussed in Section 3.1. If the percentage of stalls is less compared with the computation part, there is more chance that the benchmark speedup is linear. It is easy to observe that both bt.A, ft.B, ep.C, and lu.B exhibit only small percentage of stall cycles in the whole execution. The speedup of all the benchmarks can be observed from Figure 3 and Figure 4. At the configuration where all eight cores are used, ep.C reaches highest speedup factor, which is 7.60 , followed by is.C, which shows 6.45 . ft.B and lu.B, however, only speedup 5.63 and 5.45, respectively, if all the eight cores 


\begin{tabular}{|c|c|c|c|c|c|}
\hline Conf & Ctgy & $\alpha$ & $\beta$ & $\gamma$ & $\epsilon$ \\
\hline \multirow{2}{*}{$(1,2)$} & 1 & -1422.02 & 16638.10 & 1.15 & $-3.37 \mathrm{E}+10$ \\
& 2 & -1362.78 & 17921.22 & 1.02 & $-2.36 \mathrm{E}+10$ \\
\hline \multirow{2}{*}{$(1,4)$} & 1 & 118181.23 & -99645.46 & 0.35 & $6.80 \mathrm{E}+10$ \\
& 2 & -7743.71 & 179178.05 & -0.35 & $-3.64 \mathrm{E}+11$ \\
\hline \multirow{2}{*}{$(2,2)$} & 1 & 6327.38 & -6849.06 & 0.95 & $4.30 \mathrm{E}+09$ \\
& 2 & -921.27 & 22574.88 & 0.72 & $-4.80 \mathrm{E}+10$ \\
\hline \multirow{2}{*}{$(2,4)$} & 1 & 55261.26 & -46362.25 & 0.70 & $3.28 \mathrm{E}+10$ \\
& 2 & -5828.06 & 130347.17 & -0.11 & $-2.78 \mathrm{E}+11$ \\
\hline \multirow{2}{*}{$(2,8)$} & 1 & 186966.68 & -149787.96 & 0.11 & $8.73 \mathrm{E}+10$ \\
& 2 & -7333.23 & 154658.47 & 0.42 & $-2.68 \mathrm{E}+11$ \\
\hline
\end{tabular}

Table 5: Parameters obtained for the speedup model.

\begin{tabular}{|c|c|c|c|}
\hline Benchmark & Eexcuted_Cycles & Stall_Cycles & Percentage of stall \\
\hline bt.A & 205174317772 & 24536512493 & $11 \%$ \\
\hline ft.B & 168308642166 & 18113150861 & $10 \%$ \\
\hline cg.C & 591537183631 & 493396017764 & $45 \%$ \\
\hline ep.C & 632521811986 & 168804009044 & $21 \%$ \\
\hline is.C & 66076806330 & 53651429567 & $45 \%$ \\
\hline lu.B & 587449954695 & 132446913274 & $18 \%$ \\
\hline sp.B & 496368059294 & 154413417994 & $24 \%$ \\
\hline
\end{tabular}

Table 6: Percentage of stall in the configuration of $(2,2)$.

are used. The reason is because these two benchmarks show higher LLC references and LLC misses rates, which are 12 times and 2.5 times on average compared with ep.C and bt.A. As a result, the speedup is limited even though the percentage of stall is only approximately $20 \%$ in the configuration of $(2,2)$.

As Figure 3 shows, in general the prediction results are more accurate when there are less threads. If all eight cores are used, the average prediction error is $12 \%$ compared with the average prediction error in the configuration $(1,2)$ is $8 \%$. The serial portion of ferret workload has great variation when all 8 cores are enabled. Specifically, the average waiting time for each thread increases from around 2 seconds to 10 seconds for this particular configuration. The reason is probably because the interplay of condition variables. Regarding raytrace benchmark, it has a large portion of serial workload, around 90 seconds, which is the reason why the speedup is restricted. 

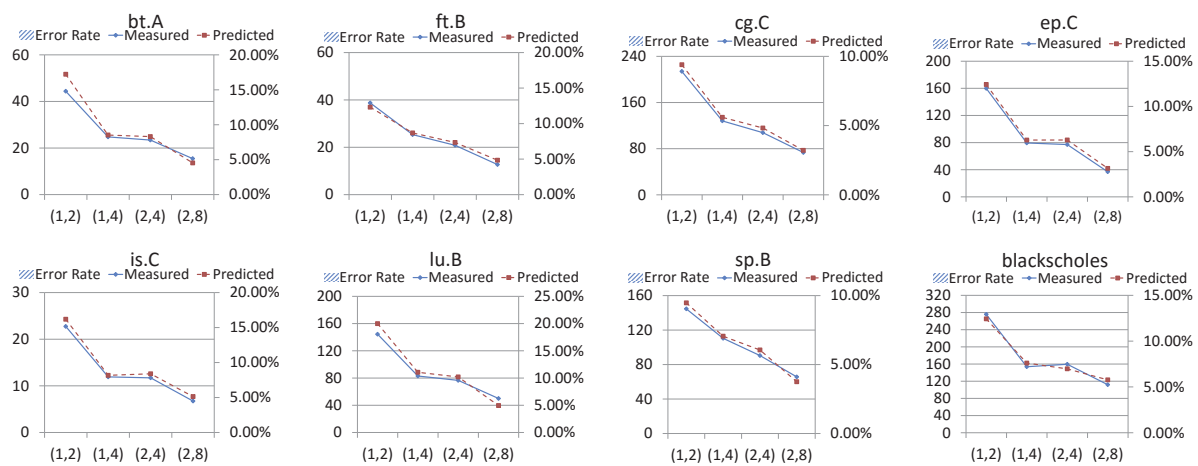

blackscholes
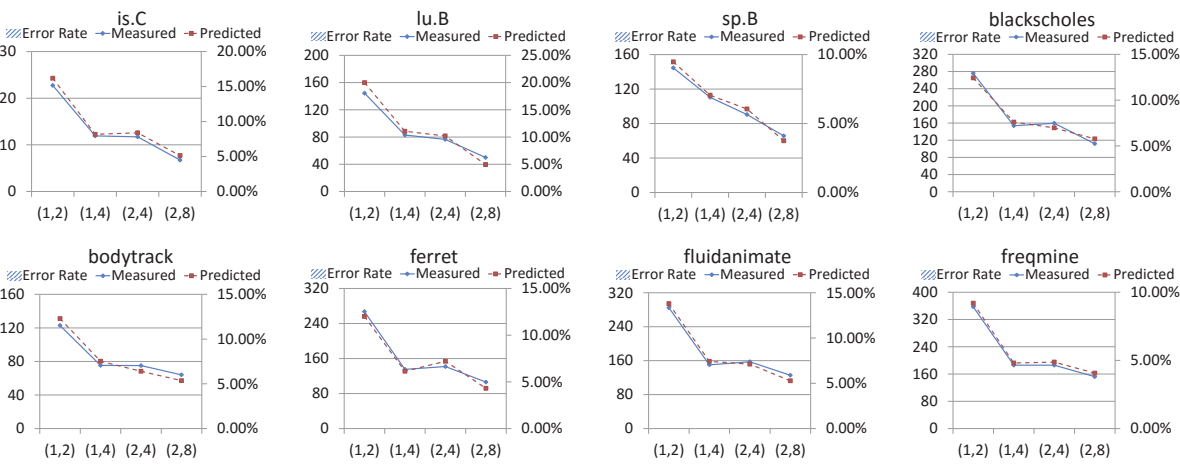

freqmine
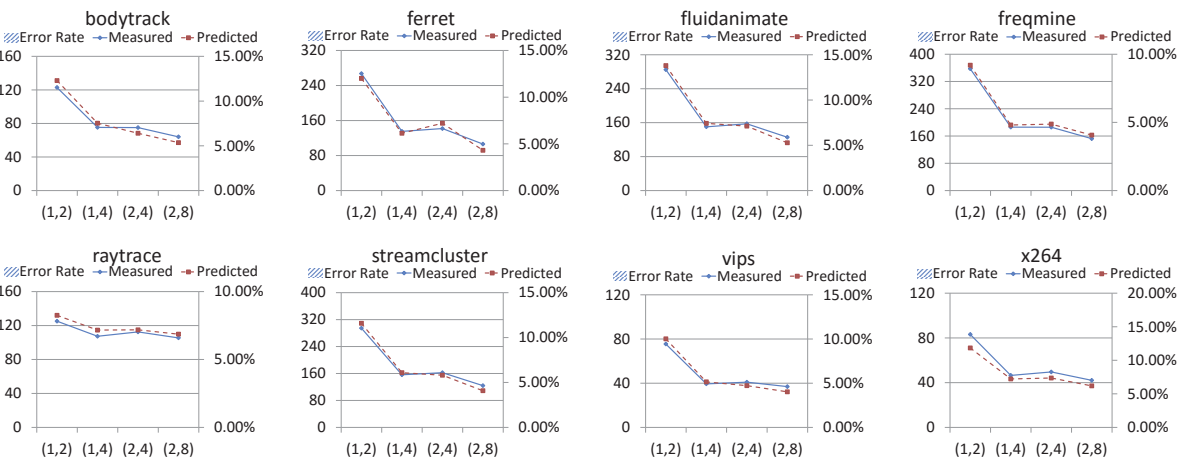

Figure 3: Measured execution time vs. predicted execution time. The unit is in second. X-axis represents each configuration. For example, (1,2) stands for one processor and totally two threads are used.

\subsection{Power Model Evaluation}

Power estimation is shown in Figure 5. The estimation of power dissipation is a challenging task because of the following reasons: first, the model that we propose does not consider the other components such as motherboards. Those parts are not the major concern of this paper. However, we measure the whole system power; second, the PMCs used in the model are limited. Basically, only four hardware counter registers are available on Xeon E5620 to be sampled simultaneously. The power model uses four of them, namely LLC references, LLC misses, executed cycles, and uops issued. The average prediction error is $8 \%$. We are able to control the prediction error rate to a relative low level by using only four counters in the prediction model because the idle power for the platform is about $143 \mathrm{~W}$, which is a large portion of the whole system. In addition, since serial portion of the workload 


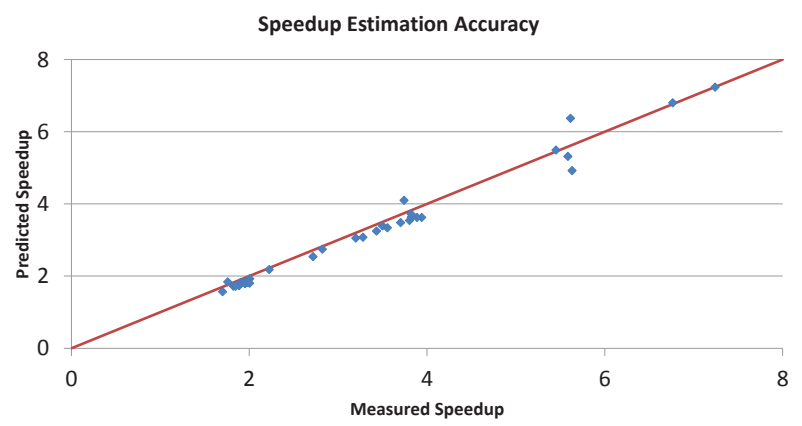

Figure 4: The predicted speedup factor using different concurrency levels and thread mapping strategies.

occupies a certain amount of the the power dissipation for PARSEC benchmarks, they consumes less power compared with that of NPB benchmarks and are less complicated to predict.

Combining execution time prediction and power dissipation prediction, we are able to calculate the total predicted energy and then determine the most energy efficient configuration. From Figure 6, we can observe that our prediction model is able to accurately predict the most energy efficient configuration for each benchmark, which for most of the tested benchmarks is the configuration that uses all eight cores. This result is different from the observation in [11]. The reason is because E5620 uses a shared L3 cache that handles most of the data access of four cores allocated on one socket. This design reduces LLC misses and increases the throughput of the whole system. The extra power dissipation added by this part is negligible compared with the performance gain. One exception is raytrace benchmark. Increasing the number of threads does not have great impact on the speedup and adds extra energy for the execution because the serial portion of the workload dominates. As a result, using four threads and one processor can produce optimal energy efficiency. However, if Intel Hyper-Threading Technique is enabled and all 16 logical cores are available, we can observe that the benefit diminishes. For FT, SP and vips benchmarks, using more logical cores do not reduce total energy consumption. But including simultaneous multithreading in the model is our future work since some of the PMCs can only measure per core events, which makes the training process obscure. 

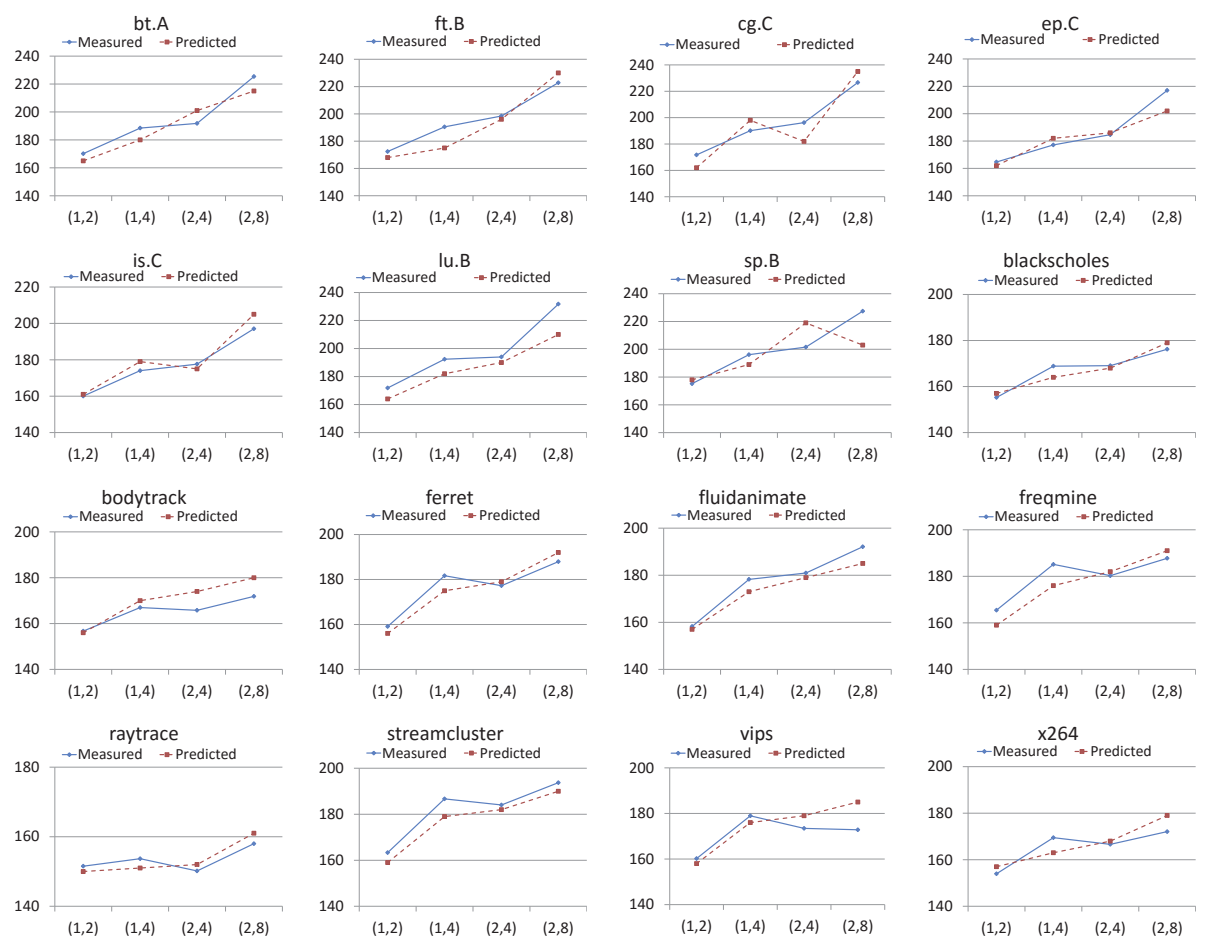

Figure 5: Measured power dissipation vs. predicted power dissipation. The unit is in watt. $\mathrm{X}$-axis represents one configuration. For example, $(1,2)$ stands for one processor and totally two threads are used.

\subsection{Run-time DVFS Evaluation}

Based on the prediction model in the previous section, we are able to select the optimal configuration for the benchmarks. In the next step, we use runtime PMCs information to predict program phases and select an appropriate frequency level for each phase. Normally, DVFS is only available for a entire processor if only one power domain is designed for the processor.

Figure 7 illustrates the additional Energy-Delay Product (EDP) obtained from the the run-time DVFS scheme. The results obtained from the first step uses the maximum CPU frequency. By applying a run-time DVFS scheme we are able to achieve additional energy savings. CG is the most memory-bounded benchmark in the test. It has the largest LLC reference rate and LLC miss rate. The total EDP obtained for CG benchmark is around $24 \%$. On the contrary, no additional saving can be obtained for EP 

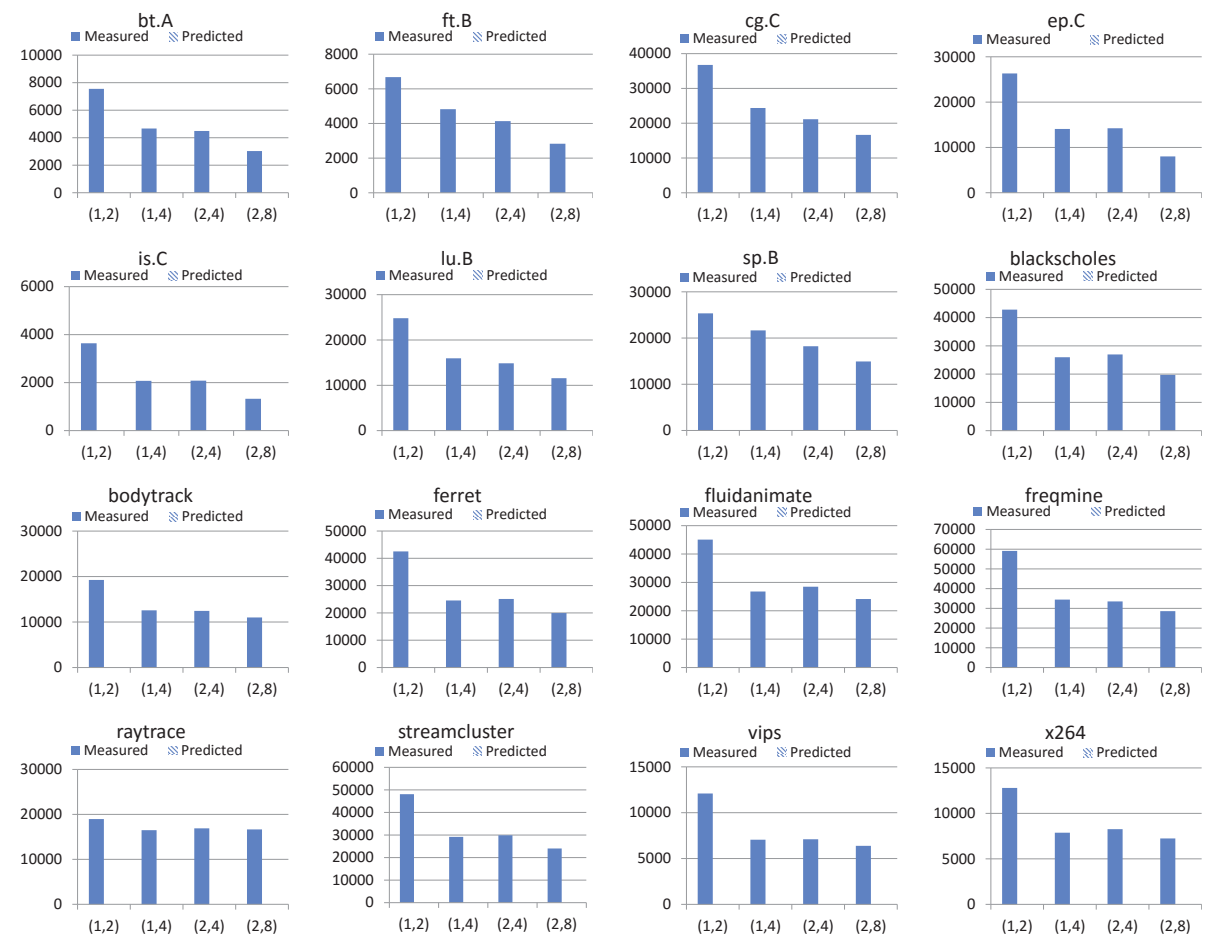

Figure 6: Measured energy consumption vs. predicted energy consumption. The unit is in Joule. $\mathrm{X}$-axis represents one configuration. For example, $(1,2)$ stands for one processor and totally two threads are used.

benchmark. freqmine benchmark produces maximum EDP saving among PARSEC benchmarks. The reason is because it has a large working set size and frequent data sharing, which produces CPU slacks. The average percentage of EDP saving is $10 \%$. Though there is more CPU idle time during the execution of PARSEC benchmarks, for example, there is as much as 90 second idle time for a CPU when executing raytrace benchmark, the energy savings achieved by using DVFS is limited. The reason is because DVFS does not impact power dissipation much during the CPU idle. For instance, the system idle power is around $137 \mathrm{~W}$ for the tested platform when it either operates at its maximum or minimum frequency. Another method that guides CPUs to enter a deeper sleep mode is needed in order to achieve more energy savings for similar benchmarks.

In order to compare the proposed DVFS scheme and the optimal solution, 
we apply a brute-force approach to obtain the optimal voltage/frequency for each phase by applying all possible settings. Because we set the sample interval relative large, the number of phases is limited. Figure 8 shows the EDP of different benchmarks using the proposed scheme compared with the optimal settings. ep.C benchmark is distinguished from others because the phases are very easy to predict and simply setting the CPU frequency to maximum produces the most energy efficiency solution. Other benchmarks exhibit different characteristics, for example, FT benchmark has various phases and exhibits to be memory-bounded in most of its phases (it has the third largest LLC miss rate, which is 3785337 misses/sec). Phases change frequently in FT benchmark. As a result, FT benchmark spends most of its execution time in the maximum frequency level. CG benchmark, although has the largest LLC reference and LLC miss rate, is supposed to spends most of the time on the second minimum frequency, which is not captured by our proposed model. The prediction model simply sets the frequency of each phase in CG benchmark to minimum frequency all the time. The average gap between the results produced by our approach to the optimal solution is around $5 \%$.

The inaccuracy of the prediction model stems from the following causes. First, the phase prediction model is relatively simple in our implementation. We use a last value prediction algorithm, which performs well if the phase of a workload is stable. Second, one of the assumptions in our two step scheme is that the LLC and memory bandwidths are independent from the CPU operating frequency and the number of threads. In reality, a slight drop in bandwidth occurs on the target platform [32].

\section{Related work}

Different speedup models are proposed to analyze the architecture and program insights of multi-core systems. Kim et al. propose an approach that predicts potential speedup from sequential execution [5]. Theoretical analysis of speedup of workloads on modern symmetric and asymmetric is provided in [33]. However, those works do not consider the problem from an energy efficiency perspective. Power aware environment is considered in building speedup models recently. Ge and Cameron [7] propose a poweraware speedup model that is derived from Amdahls Law [34]. The proposed scheme divides parallel workload into two major parts, which include on-chip and off-chip parts. In addition, each one of them can be categorized as two 


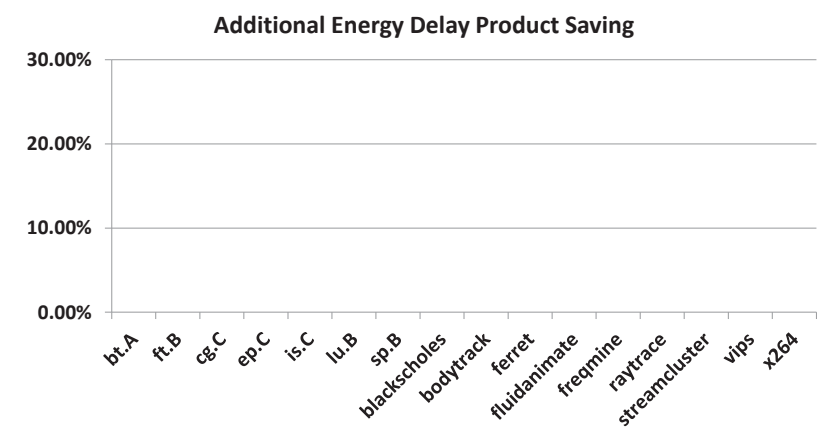

Figure 7: Additional EDP using run-time DVFS when the optimal configuration generated from the first step is used. The results are compared to the setting that uses maximum frequency only.

subclasses, one of which is affected by DVFS and the other is not. However, the authors do not predict speedup practically based on the model.

Combining power aware features of a system and parallel workload characteristics, Curtis-Maury et al. proposed a multi-dimensional prediction model that uses DCT and DVFS. This work is similar to our approach [11]. However, the method is based on empirical models to predict concurrency level that lack architectural insights, which limit the applicability of the proposed model. For example, different micro-architecture might use different empirical parameters. Ge et al. propose an analytical model to analyze the energy efficiency issue using a speedup and power model [13]. The proposed work is verified by case studies. The major difference between the proposed work and [13] is that they focus on analysis while we focus on prediction. Saravanan et al. simulates different power features according to the processor characteristics, such as out-of-order execution [35]. However, they did not consider the workloads and their requirements. Li et al. propose SolarCore, a solar energy driven, multi-core architecture power management scheme that combines maximal power provisioning control and workload run-time optimization[36]. Their system is capable of achieving the optimal operation condition of solar panels, however, they are optimized for solar energy system and we are aiming at a more general environment. Cochran et al. proposes Pack \& Cap, a control technique designed to make optimal DVFS and thread packing control decisions in order to maximize performance within a power budget[37]. They requires an offline selection of over 200 counters, our model 

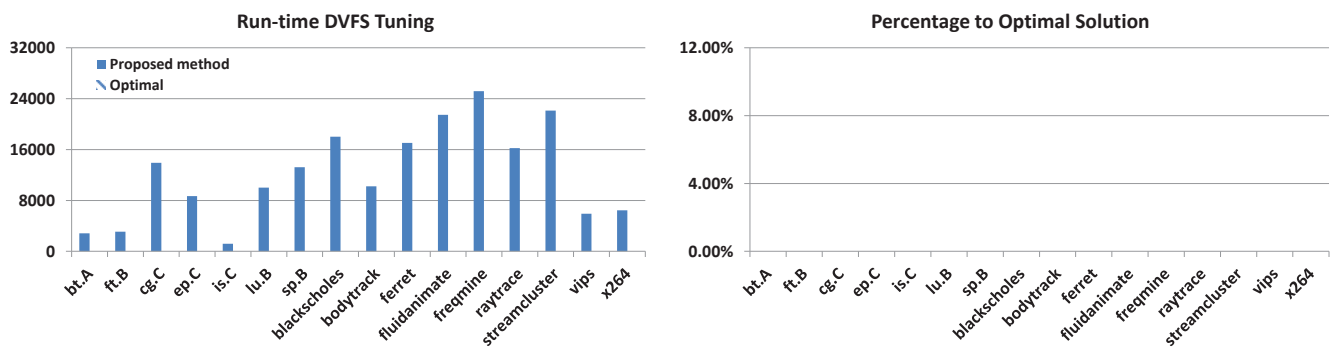

Figure 8: Comparing the proposed run-time DVFS adjusting algorithm to the optimal solution in terms of EDP saving. Optimal solution uses an offline brute-force approach to obtain the optimal frequency for each phase.

is based on a set of pre-selected parameters (IPC, LLC_R, LLC_M, Stall) from an analytical models. The process of training is greatly reduced.

Estimating power dissipation using PMCs is one of the most important topics in HPC because the estimated results can be used for peak power control and thermal management. Joseph and Martonosi propose one of the earliest works on estimating power dissipation using Performance Monitoring Counters (PMCs) [38]. Goel et al. propose a method to [39] estimate system power dissipation of different architectures. The results show that applying a different set of PMCs according to architectural characteristics produces better estimation accuracy. These methods, however, do not consider the energy efficiency of a workload.

Adjusting DVFS according to program phases is an effective method to reduce energy consumption given a performance metric. One of the most effective methods is scaling down the CPU voltage and frequency during the memory intensive phases. Isci et al. introduce a prediction model of a memory intensive phases during a program's execution [29]. Spiliopoulos et al. propose a Green Governor that utilizes the slacks in memory-bounded applications to save energy with limited performance loss [40]. The key difference between the proposed approach and the aforementioned approaches is that our approach concerns the scalability of workloads.

Tuning memory modes to achieve memory savings is also considered to be an effective way. Deng et al. propose MemScale which enables DVFS on Memory Controller and DFS on Memory Channels to explore dynamic energy saving [41]. Other than using dynamice tuning, Liu et al. changes 
memory refresh rate of less important data in memory while keeping regular refreshing rate for important data [42] to achieve energy saving. Wu et al. propose a bank level controller for memory subsystem that predicts data locality and groups relevant pages togather [43]. However, the hardware techniques on the memory subsystem are not available yet.

\section{Summary and Future Work}

In this paper, we propose a practical analytical prediction model that produces energy efficient configurations for parallel workloads. The first step of the model uses execution information of configuration $(2,2)$ and system architecture information to produce the optimal concurrency level and thread mapping strategy by predicting the potential speedup and average power dissipation. DVFS technique is used in the second step to adjust CPU voltage/frequency at run-time to further reduce the energy consumption. NPB OMP and PARSEC benchmark suites are used to evaluate the proposed work. The experimental results based on a Intel E5620 platform shows that the proposed model is able to accurately predict the optimal energy efficiency configuration in the first step. The second step further reduces energy consumption for the configuration obtained from the first step. The average EDP saving is 10\%. An off-line optimal solution calculated to compare with the proposed scheme shows that the average extra EDP obtained by optimal solution is within $5 \%$.

As the inception of dark silicon phenomenon, energy efficient application execution prediction becomes more and more important. A nature next step is to consider multiple applications scenario, and heterogeneous multicore scenarios. The current work does not consider the Simultaneous Multithreading (SMT) For example, if Hyper-Threading is enabled on Xeon E5620, the speedup model and power model need to be revisited.

\section{Acknowledgement}

This work is in part supported by NSF grant CNS-1205338. This material is based upon work supporting while serving at the National Science Foundation. This work in-part used the resources provided by the Oak Ridge Leadership Computing Facility at the Oak Ridge National Laboratory, which is managed by UT Battelle, LLC for the U.S. DOE (under the contract No. DE-AC05-00OR22725). 
[1] D. Meisner, C. M. Sadler, L. A. Barroso, W.-D. Weber, T. F. Wenisch, Power management of online data-intensive services, in: Proceeding of the 38th annual international symposium on Computer architecture, ISCA '11, ACM, New York, NY, USA, 2011, pp. 319-330.

[2] N. Sharma, S. Barker, D. Irwin, P. Shenoy, Blink: managing server clusters on intermittent power, in: Proceedings of the sixteenth international conference on Architectural support for programming languages and operating systems, ASPLOS XVI, ACM, New York, NY, USA, 2011, pp. $185-198$.

[3] H. Esmaeilzadeh, E. Blem, R. S. Amant, K. Sankaralingam, D. Burger, Power challenges may end the multicore era, Commun. ACM 56 (2013) 93-102.

[4] S. Wang, W. Shi, CPT: An Energy Efficiency Model for Multi-core Computer Systems, Technical Report MIST-TR-2012-008, Wayne State University, 2012.

[5] M. Kim, P. Kumar, H. Kim, B. Brett, Predicting potential speedup of serial code via lightweight profiling and emulations with memory performance model, in: Parallel Distributed Processing Symposium (IPDPS), 2012 IEEE 26th International, pp. 1318 -1329.

[6] C. Hughes, T. Li, Optimizing throughput/power trade-offs in hardware transactional memory using dvfs and intelligent scheduling, in: Proceedings of the international conference on Supercomputing, ICS '11, ACM, New York, NY, USA, 2011, pp. 141-150.

[7] R. Ge, K. Cameron, Power-aware speedup, in: Parallel and Distributed Processing Symposium, 2007. IPDPS 2007. IEEE International, pp. 1 $-10$.

[8] T. Cao, S. M. Blackburn, T. Gao, K. S. McKinley, The yin and yang of power and performance for asymmetric hardware and managed software, in: Proceedings of the 39th Annual International Symposium on Computer Architecture, ISCA '12, IEEE Computer Society, Washington, DC, USA, 2012, pp. 225-236.

[9] S. Eggers, T. Jeremiassen, Eliminating false sharing, University of Washington, Department of Computer Science, 1990. 
[10] D. Ziakas, A. Baum, R. A. Maddox, R. J. Safranek, Intel ${ }^{\circledR}$; quickpath interconnect architectural features supporting scalable system architectures, in: Proceedings of the 2010 18th IEEE Symposium on High Performance Interconnects, HOTI '10, IEEE Computer Society, Washington, DC, USA, 2010, pp. 1-6.

[11] M. Curtis-Maury, A. Shah, F. Blagojevic, D. S. Nikolopoulos, B. R. de Supinski, M. Schulz, Prediction models for multi-dimensional powerperformance optimization on many cores, in: Proceedings of the 17th international conference on Parallel architectures and compilation techniques, PACT '08, ACM, New York, NY, USA, 2008, pp. 250-259.

[12] L. Niccolini, G. Iannaccone, S. Ratnasamy, J. Chandrashekar, L. Rizzo, Building a power-proportional software router, in: Proceedings of the 2012 USENIX conference on Annual Technical Conference, USENIX ATC'12, USENIX Association, Berkeley, CA, USA, 2012, pp. 8-8.

[13] R. Ge, X. Feng, K. Cameron, Modeling and evaluating energyperformance efficiency of parallel processing on multicore based power aware systems, in: Parallel Distributed Processing, 2009. IPDPS 2009. IEEE International Symposium on, pp. $1-8$.

[14] C. Carothers, K. Perumalla, R. Fujimoto, The effect of state-saving in optimistic simulation on a cache-coherent non-uniform memory access architecture, in: Simulation Conference Proceedings, 1999 Winter, volume 2, pp. $1624-1633$ vol.2.

[15] S. Hong, S. Narayanan, M. Kandemir, O. Ozturk, Process variation aware thread mapping for chip multiprocessors, in: Design, Automation Test in Europe Conference Exhibition, 2009. DATE '09., pp. 821 -826.

[16] M. Castro, L. Goes, C. Ribeiro, M. Cole, M. Cintra, J.-F. Mehaut, A machine learning-based approach for thread mapping on transactional memory applications, in: High Performance Computing (HiPC), 2011 18th International Conference on, pp. $1-10$.

[17] A. Gautham, K. Korgaonkar, P. Slpsk, S. Balachandran, K. Veezhinathan, The implications of shared data synchronization techniques on multi-core energy efficiency, in: Proceedings of the 2012 USENIX conference on Power-Aware Computing and Systems, HotPower'12, USENIX Association, Berkeley, CA, USA, 2012, pp. 6-6. 
[18] J. A. Joao, M. A. Suleman, O. Mutlu, Y. N. Patt, Bottleneck identification and scheduling in multithreaded applications, in: Proceedings of the seventeenth international conference on Architectural Support for Programming Languages and Operating Systems, ASPLOS XVII, ACM, New York, NY, USA, 2012, pp. 223-234.

[19] C. Bienia, K. Li, Parsec 2.0: A new benchmark suite for chipmultiprocessors, in: Proceedings of the 5th Annual Workshop on Modeling, Benchmarking and Simulation.

[20] Intel Corporation, Intel ${ }^{\circledR} 64$ and IA-32 Architectures Software Developer's Manual, 253669-033US, 2009.

[21] David Levinthal, Performance Analysis Guide for Intel ${ }^{\circledR}$ Core $^{\mathrm{TM}}$ i7 Processor and Intel ${ }^{\circledR}$ Xeon $^{\mathrm{TM}} 5500$ processors, 2010.

[22] R. E. Bryant, D. R. O'Hallaron, Computer Systems: A Programmer's Perspective, Addison-Wesley Publishing Company, USA, 2010.

[23] S. Wang, H. Chen, W. Shi, SPAN: A software power analyzer for multicore computer systems, Sustainable Computing: Informatics and Systems 1 (2011) $23-34$.

[24] T. Li, L. K. John, Run-time modeling and estimation of operating system power consumption, SIGMETRICS Perform. Eval. Rev. 31 (2003) $160-171$.

[25] R. Bertran, M. Gonzalez, X. Martorell, N. Navarro, E. Ayguade, Decomposable and responsive power models for multicore processors using performance counters, in: Proceedings of the 24th ACM International Conference on Supercomputing, ICS '10, ACM, New York, NY, USA, 2010, pp. 147-158.

[26] C. Isci, M. Martonosi, Runtime power monitoring in high-end processors: Methodology and empirical data, in: Proceedings of the 36th annual IEEE/ACM International Symposium on Microarchitecture, MICRO 36, IEEE Computer Society, Washington, DC, USA, 2003.

[27] D.-C. Juan, D. Marculescu, Power-aware performance increase via core/uncore reinforcement control for chip-multiprocessors, in: Proceedings of the $2012 \mathrm{ACM} / \mathrm{IEEE}$ international symposium on Low power 
electronics and design, ISLPED '12, ACM, New York, NY, USA, 2012, pp. $97-102$.

[28] R. Z. Ayoub, U. Ogras, E. Gorbatov, Y. Jin, T. Kam, P. Diefenbaugh, T. Rosing, Os-level power minimization under tight performance constraints in general purpose systems, in: Proceedings of the 17th IEEE/ACM international symposium on Low-power electronics and design, ISLPED '11, IEEE Press, Piscataway, NJ, USA, 2011, pp. 321-326.

[29] C. Isci, G. Contreras, M. Martonosi, Live, runtime phase monitoring and prediction on real systems with application to dynamic power management, in: Microarchitecture, 2006. MICRO-39. 39th Annual IEEE/ACM International Symposium on, pp. $359-370$.

[30] C. Isci, M. Martonosi, Phase characterization for power: evaluating control-flow-based and event-counter-based techniques, in: HighPerformance Computer Architecture, 2006. The Twelfth International Symposium on, pp. $121-132$.

[31] W. de Bruijn, D. Truong, J. Brandeburg, F. Weisbecker, C. R. Mafra, P. Enberg, Perf Wiki, https://perf.wiki.kernel.org/, 2009.

[32] R. Schöne, D. Hackenberg, D. Molka, Memory performance at reduced cpu clock speeds: an analysis of current x86_64 processors, in: Proceedings of the 2012 USENIX conference on Power-Aware Computing and Systems, HotPower'12, Berkeley, CA, USA, pp. 9-9.

[33] E. Yao, Y. Bao, G. Tan, M. Chen, Extending amdahl's law in the multicore era, SIGMETRICS Perform. Eval. Rev. 37 (2009) 24-26.

[34] G. M. Amdahl, Validity of the single processor approach to achieving large scale computing capabilities, in: Proceedings of the April 18-20, 1967, spring joint computer conference, AFIPS '67 (Spring), ACM, New York, NY, USA, 1967, pp. 483-485.

[35] V. Saravanan, S. K. Chandran, S. Punnekkat, D. P. Kothari, A study on factors influencing power consumption in multithreaded and multicore cpus, W. Trans. on Comp. 10 (2011) 93-103.

[36] C. Li, W. Zhang, C.-B. Cho, T. Li, Solarcore: Solar energy driven multicore architecture power management, in: High Performance Computer 
Architecture, 2011 IEEE 17th International Symposium on, pp. 205216.

[37] R. Cochran, C. Hankendi, A. K. Coskun, S. Reda, Pack \& cap: Adaptive dvfs and thread packing under power caps, in: Proceedings of the 44th Annual IEEE/ACM International Symposium on Microarchitecture, MICRO-44, ACM, New York, NY, USA, 2011, pp. 175-185.

[38] R. Joseph, M. Martonosi, Run-time power estimation in high performance microprocessors, in: Proceedings of the 2001 international symposium on Low power electronics and design, ISLPED '01, ACM, New York, NY, USA, 2001, pp. 135-140.

[39] B. Goel, S. McKee, R. Gioiosa, K. Singh, M. Bhadauria, M. Cesati, Portable, scalable, per-core power estimation for intelligent resource management, in: Green Computing Conference, 2010 International, pp. $135-146$.

[40] V. Spiliopoulos, S. Kaxiras, G. Keramidas, Green governors: A framework for continuously adaptive dvfs, in: Green Computing Conference and Workshops (IGCC), 2011 International, pp. 1 -8.

[41] Q. Deng, D. Meisner, L. Ramos, T. F. Wenisch, R. Bianchini, Memscale: active low-power modes for main memory, in: Proceedings of the sixteenth international conference on Architectural support for programming languages and operating systems, ASPLOS XVI, ACM, New York, NY, USA, 2011, pp. 225-238.

[42] S. Liu, K. Pattabiraman, T. Moscibroda, B. G. Zorn, Flikker: saving dram refresh-power through critical data partitioning, in: Proceedings of the sixteenth international conference on Architectural support for programming languages and operating systems, ASPLOS XVI, ACM, New York, NY, USA, 2011, pp. 213-224.

[43] D. Wu, B. He, X. Tang, J. Xu, M. Guo, Ramzzz: rank-aware dram power management with dynamic migrations and demotions, in: Proceedings of the International Conference on High Performance Computing, Networking, Storage and Analysis, SC '12, IEEE Computer Society Press, Los Alamitos, CA, USA, 2012, pp. 32:1-32:11. 


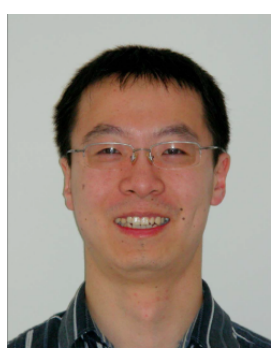

Shinan Wang holds a Ph.D. degree in Computer Science of Wayne State University (2014). His research interests include computer system power profiling and management. His research results have been published in Sustainable Computing: Informatics and Systems, ICEAC, WEED, and several other conferences and journals. He is a recipient of IPSN 2009 and IGCC 2013 student's travel grants. He is also awarded with Wayne State University Summer Dissertation Fellowship.

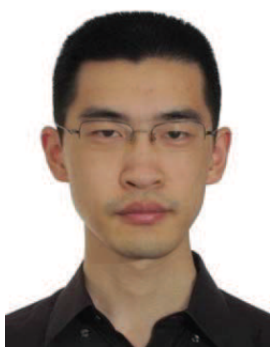

Bing Luo received the BSc degree in Information and Computing Science from East China University of Science and Technology, Shanghai, China. He is currently working toward the PhD degree in computer science at Wayne State University, Detroit, Michigan. His research interests include operating systems, artificial intelligence, and energy efficient computing system. He is a recipient of ISCA 2013 student's travel grants. He is a student member of the IEEE.

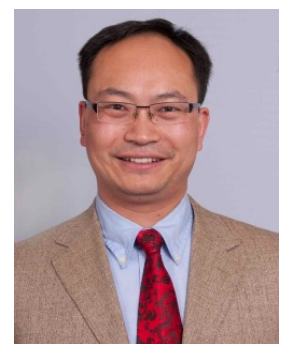

Weisong Shi is a professor of computer science at Wayne State University, where he leads the Mobile and Internet Systems Laboratory. He received his B. E. from Xidian University in 1995, and Ph.D. from the Chinese Academy of Sciences in 2000, both in Computer Engineering. His research interests include parallel and Internet computing, energy-efficient computer systems, mobile computing and smart health. Dr. Shi has published over 140 peer-reviewed journal and conference papers and has an $\mathrm{H}$-index of 30 . He is the chair of the IEEE CS Technical Committee on the Internet, and serves on the editorial board of IEEE Internet Computing, Elsevier Sustainable Computing, Journal of Computer Science and Technology (JCST) and International Journal of Sensor Networks. He was a recipient of National Outstanding PhD dissertation award of China (2002) and the NSF CAREER award (2007), Wayne State University Career Development Chair award (2009), and the Best Paper award of ICWE'04, IEEE IPDPS'05, HPCChina'12 and IEEE IISWC'12. He is a senior member of the IEEE and ACM, a member of the USENIX.

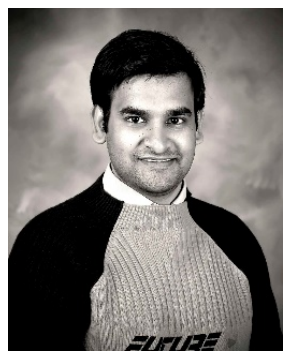

Devesh Tiwari is a Research Scientist in the Oak Ridge Leadership Computing Facility at the Oak Ridge National Laboratory. He primarily focuses on making the large-scale computing facilities more energy efficient and reliable while expediting the process of scientific discovery. His research interests are modeling, evaluating and simulating large-scale computer systems' performance, power-efficiency, and reliability. Devesh graduated with a bachelors degree in Computer Science and Engineering from Indian 
Institute of Technology (IIT Kanpur), India, and got the PhD degree in Computer Engineering from North Carolina State University. His papers have earned best paper award nominations at Supercomputing (SC), DSN, and IPDPS conferences. He has also been invited to serve on the program committees of several computer systems conferences such as ICDCS, SC, IPDPS, ISPASS, and CCGrid. 